\title{
Effect of Streptozotocin on Liver Histology Damage in Rats Model of Gestational Diabetes Mellitus
}

\author{
Liganda Endo Mahata ${ }^{1}$, Hirowati $\mathrm{Ali}^{2}$, Arina Widya Murni ${ }^{3}$ \\ ${ }^{1}$ Department of Pharmacology and Therapy, Faculty of Medicine, Universitas Andalas, Indonesia \\ ${ }^{2}$ Department of Biochemistry, Faculty of Medicine, Universitas Andalas, Padang, Indonesia \\ ${ }^{3}$ Department of Internal Medicine, Faculty of Medicine, Universitas Andalas, Padang, Indonesia
}

Corresponding Author: Liganda Endo Mahata

\begin{abstract}
Background: Gestational Diabetes Mellitus (GDM) is a disorder of carbohydrate metabolism that causes hyperglycemia, insulin resistance and failure of organs especially the liver. There is great interest in understanding the pathophysiology and treatment of GDM. Due to ethical issues involving human studies, it is necessary to use animal models to understand pathophysiology and potential treatment for GDM. Streptozotocin-induced diabetes mellitus in pregnant rats was commonly used by several author.
\end{abstract}

Aims: The aim of this study is to investigate the effect of streptozotocin (STZ) on liver histology in pregnant rats.

Methods: Pregnant rats were divided into two groups; 1) Negative control, 2) Positive control. Positive control were pregnant rats induced with a single intraperitoneal injection of streptozotocin $40 \mathrm{mg} / \mathrm{Kg}$ b.w. Fourteen days after induction, rats were sacrificed to evaluate the histopathological effect of STZ on the liver using hematoxylin Eosin staining and calculate the presentation of degraded cell and sinusoidal area with ImageJ $1.49 \mathrm{v}$ software, National Institute of Health, Bethesda, MD, USA. Data were processed statistically using SPSS with TTest.

Results: Microscopic examination of the liver of STZ-induced rats showed histologic changes in the form of an increase in the number of degenerated cells and a significant expansion of the sinusoidal area $(\mathrm{p}<0.000)$. The percentage of degenerated cells in the healthy group was $9.3 \%$, increased to $70 \%$ in the STZ-induced group. In addition, the percentage of the sinusoidal area, which was $19.98 \%$ in the healthy group, increased to $49.5 \%$

Conclusions: Streptozotocin induces liver damage in the pregnant rats model.

Keywords: Gestational Diabetes Mellitus, Streptozotocin, Liver, Histology

\section{INTRODUCTION}

Gestational diabetes mellitus (GDM) is a disorder of carbohydrate metabolism that causes hyperglycemia during pregnancy. This disease is the most common metabolic disease during pregnancy and has a negative impact due to complication from GDM. Pregnancy under diabetic conditions is complicated by a number of possibly leading to abnormalities during fetal and embryonic development. $[1,2]$

There is great interest in understanding the pathophysiology and treatment of GDM. Due to ethical issues involving human studies, it is necessary to use animal models to understand pathophysiology and potential treatment for GDM. Streptozotocin-induced diabetes mellitus in pregnant rats was commonly used by several authors. Streptozotocin is an antimicrobial drug used as a chemotherapy drug. ${ }^{[3]}$ This drug can cause specific necrosis in pancreatic cells so that it becomes the leading choice to form a rats model of hyperglycemia ${ }^{[4]}$. Streptozotocin induction, besides damaging pancreatic cells, also causes liver damage ${ }^{[4]}$. Previous 
studies reported liver cell damage and sinusoidal dilatation in the liver histology of streptozotocin-induced diabetes mellitus rats model. $^{[5,6]}$

However, previous publications have scarcely investigated the effects of maternal diabetes on liver histological architecture. ${ }^{[1]}$ Thus, the study aims to assess the effect of STZ-induced diabetes during pregnancy of rat histology on liver architecture.

\section{METHODS}

\section{Study design}

This study is an experimental and post-test-only control design to determine the effect of STZ on liver histology in pregnant rats. There were two groups of experimental animals in this study, consisting of negative control and positive control. The ethics committee approved the study of the faculty of medicine Andalas University (No.378/KEP/FK/2019)

\section{Planned study size}

The subjects were 12 pregnant white rats (Rattus novergicu L). The approximate weight was $180 \mathrm{gr}-250 \mathrm{gr}$. Rats acclimatized for one week. Male and female rats combined 2: 1 to induce pregnancy. Twelve confirmed pregnant rats were divided into two groups. Group 1 was the negative group and the second group was the positive group induced with $2 \%$ streptozotocin $40 \mathrm{mg} / \mathrm{kg}$ BW. ${ }^{[7]}$ GDM state obtained 72 hours postinjection, the examination of blood glucose levels $\geq 200 \mathrm{mg} / \mathrm{dl}-\leq 300 \mathrm{mg} / \mathrm{dl}$ using a blood glucometer strips

Fourteen days after the induction of streptozotocin, the rat model was sacrificed. The liver was taken to perform a Hematoxylin eosin (HE) examination. The rat liver tissue was fixed with formalin, processed into paraffin blocks with a thickness of 4 micrometers, and then stained with hematoxylin-eosin.

\section{Data collection procedure}

The cell damage proportion was assessed by taking photomicrograph images at 400x magnification in 5 fields of view. The number of apoptotic cells and sinusoidal area collected to assess cell damage percentage. Sinusoidal area calculated using Image J $(1.49 \mathrm{v}$ software, National Institute of Health, Bethesda, MD, USA)

\section{Data analysis}

The proportion of cell damage was assessed by counting the number of cells with signs of degeneration or necrosis, then compared with all cells in the field of view and reported in the form of percent values. The proportion of the sinusoidal area was reported in percent of the mean area. The research data were statistically processed using SPSS. A normality test was performed with Shapiro Wilk and continued with t-test analysis to see the normality of data distribution.

\section{RESULT}

Histologically, microscopic changes were seen between the negative and positive control groups. Histology of liver cells in the positive control group showed an increase in signs of necrosis with a fragmented nucleus and karyolysis (Fig, 1). The average liver cell damage increased between the negative group $(\mathrm{KN})$ and the positive group (KP) from $10 \%(\mathrm{KP})$ to $70 \%$ (KP) (Table 1).

Table 1: The proportion of damaged cells with degeneration/necrosis of liver cells in experimental rats.

\begin{tabular}{|l|l|l|l|}
\hline Subject group & n & Liver cell damage rate (\%) & p- value \\
\hline $\mathrm{KN}$ & 6 & 10,0 & 0,000 \\
\hline $\mathrm{KP}$ & 6 & 70,0 & \\
\hline
\end{tabular}

Assessment of tissue edema was performed using 400x magnification in 5 fields of view. Tissue edema was interpreted as a dilated and unstained sinusoidal area (Fig. 2). Liver histology in the rat model of the positive control group showed edema with sinusoidal and vascular widening that tended to increase (Table 2). 


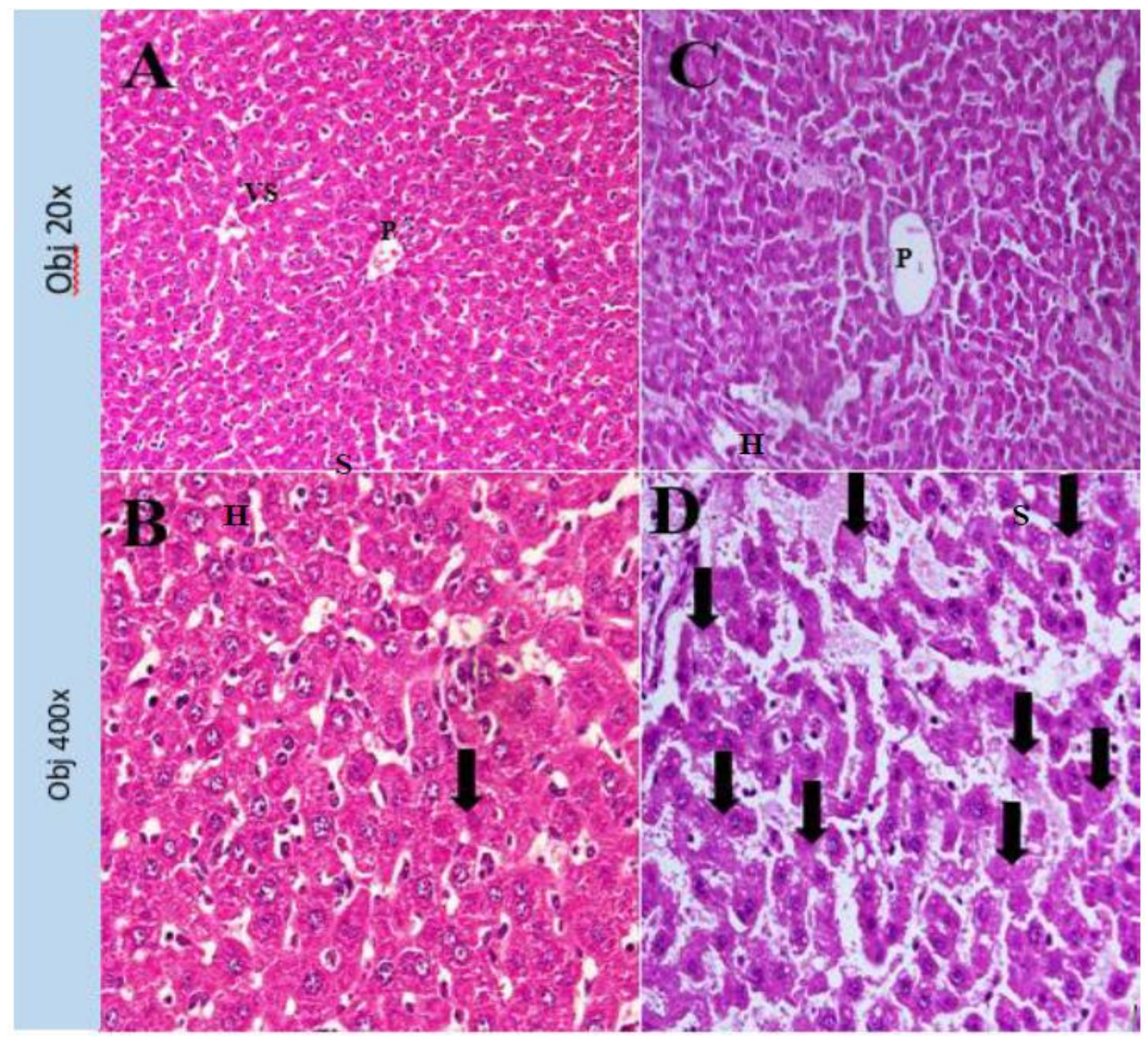

Fig.1Tissue histology of experimental animals, showing negative control animals ( $a$ and $b$ ) and animal models of gestational diabetes mellitus (c and d). Histological features of liver tissue appear to be arranged in trabeculae (H), central veins (Vs), portal veins (P), and sinusoids (S). Positive animal models show degenerated hepatocytes.
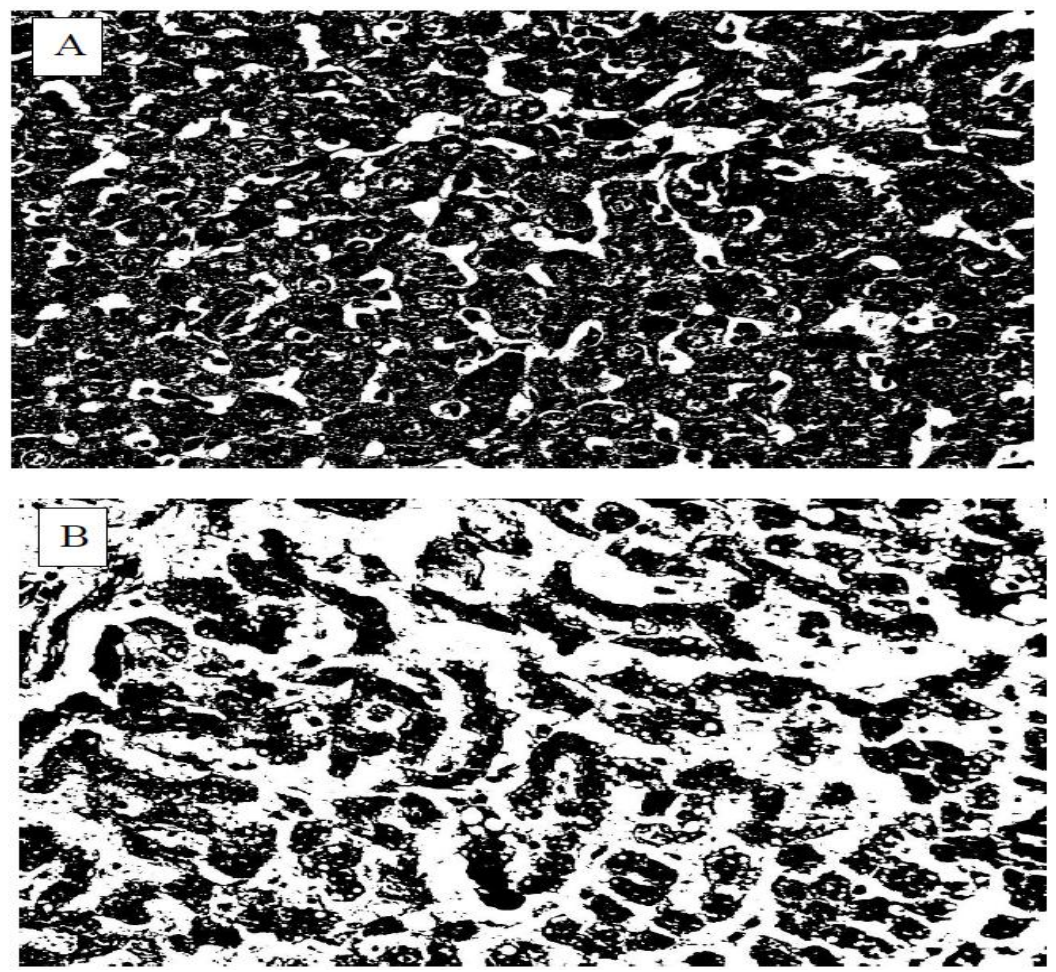

Fig.2: Measurement of liver tissue edema was performed using the ImageJ program (ImageJ 1.49v software, National Institute of Health, Bethesda, MD, USA). Hepatocytes in the trabeculae appear as black areas and sinusoids as unstained areas (empty areas). The ImageJ program measured the widening sinusoid area as the proportion of the sinusoid area to the total area, which was reported as a percent of the mean area. The negative control group (A) shows a smaller sinusoid area than the gestational diabetes mellitus rats $(\mathbf{B})$. 
Tabel 2: Table of the proportion of damaged cells with edema of liver cells in experimental rats.

\begin{tabular}{|l|l|l|l|}
\hline $\begin{array}{l}\text { Subject } \\
\text { Group }\end{array}$ & $\mathbf{n}$ & $\begin{array}{l}\text { Percentage proportion of } \\
\text { tissue edema (\%) }\end{array}$ & p- value \\
\hline KN & 6 & 20,0 & 0,000 \\
\hline KP & 6 & 49,5 & \\
\hline
\end{tabular}

\section{DISCUSSION}

The results showed that streptozotocin (STZ) induction affected liver damage in GDM rats. Streptozotocin is an antimicrobial drug used as a chemotherapy drug. This drug can cause specific necrosis in pancreatic cells so that it becomes the leading choice to form a rat model of hyperglycemia. ${ }^{[3]}$ Hyperglycemia that occurs in rats model of gestational diabetes mellitus in this study turned out to impact the liver, where it was found an increase in hepatocyte degeneration and edema in the sinusoidal liver.

The liver damage found in this study is in line with the previous study who found that the liver in STZ-induced rats showed hyperemia in the central venous area, increased sinusoidal spaces and increased necrosis in hepatic. In addition, other studies have also reported liver cell damage, sinusoidal dilatation in the central venous area, increased apoptosis of cells, and increased lipid droplets in liver cells ${ }^{[1,8]}$

Liver damage in rat models of gestational diabetes mellitus can occur due to hyperglycemia conditions in GDM, causing hyperinsulinemia and insulin resistance. Insulin resistance causes an increase in the lipolysis process so that free fatty acids will accumulate in the liver. Hyperglycemia conditions will trigger inflammatory conditions and oxidative stress, thereby worsening the process of liver damage by triggering the activation of NF-B, which will stimulate the activity of pro-apoptotic genes in liver cells and increase the production of reactive oxygen species (ROS). ${ }^{[9]}$

Oxidation stress is a condition of imbalance between the ratio of oxidants and antioxidants, causing an increase in free radicals. The liver is the main organ that plays a role in detoxification, which is equipped with potent antioxidants such as superoxide dismutase (SOD), catalase (CAT), and glutathione (GSH). Hyperglycemia conditions will reduce SOD, GSH, and CAT activity, thereby increasing oxidants such as reactive oxygen species (ROS), which contribute to liver damage ${ }^{[2]}$

\section{CONCLUSION}

In conclusion, the liver of streptozotocin-induced Diabetic Mellitus gestational rats will degenerate in the form of an increase in the percentage of cell apoptosis and tissue edema.

\section{Acknowledgement: None}

\section{Conflict of Interest: None}

\section{Source of Funding: None}

\section{Ethical Approval: Approved}

\section{REFERENCES}

1. Ahmed OM, Bas A. Maternal Diabetes Mellitus Disturbs Histological Architecture and Integrity of Liver and Kidney in Rat Offspring. Merit Res J Med Med Sci [Internet]. 2015;(August). Available from: http://www.meritresearchjournals.org/mms/i ndex.htm

2. Mohamed J, Nazratun Nafizah AH, Zariyantey AH, Budin SB. Mechanisms of diabetes-induced liver damage: The role of oxidative stress and inflammation. Sultan Qaboos Univ Med J. 2016;16(2):e132-41.

3. Akbarzadeh A, Norouzian D, Mehrabi MR, Jamshidi S, Farhangi A, Allah Verdi A, et al. Induction of diabetes by Streptozotocin in rats. Indian J Clin Biochem. 2007; 22(2):60-4.

4. Damasceno DC, Netto AO, Iessi IL, Gallego FQ, Corvino SB, Dallaqua B, et al. Streptozotocin-induced diabetes models: Pathophysiological mechanisms and fetal outcomes. Biomed Res Int. 2014;2014.

5. Kohl T, Gehrke N, Schad A, Nagel M, Wörns MA, Sprinzl MF, et al. Diabetic liver injury from streptozotocin is regulated through the caspase-8 homolog cFLIP involving activation of JNK2 and intrahepatic immunocompetent cells. Cell Death Dis. 2013;4(7). 
Liganda Endo Mahata et.al. Effect of streptozotocin on liver histology damage in rats model of gestational diabetes mellitus.

6. Carnovale CE, Garay EAR. Reversible impairment of hepatobiliary function induced by streptozotocin in the rat. Experientia. 1984;40(3):248-50.

7. Wang Y, Feng Q, Niu X, Liu X, Xu K, Yang $X$, et al. The Therapeutic Effect of Zuogui Wan in Gestational Diabetes Mellitus Rats The Therapeutic Effect of Zuogui Wan in Gestational Diabetes Mellitus Rats. 2014;(July).

8. Rodríguez V, Plavnik L, Tolosa de Talamoni N. Naringin attenuates liver damage in streptozotocin-induced diabetic rats. Biomed Pharmacother [Internet]. 2018;105(April):95-102. Available from: https://doi.org/10.1016/j.biopha.2018.05.12 0

9. Ugwu M, Umar I, Baku U, Dasonfunjo, Ukpanukpong $\mathrm{R}$, Yakubunu $\mathrm{O}$, et al. Antioxidant Status and Organ Function in Streptozotocin-Induced Diabetic Rats treated with Aqueous, Methanolic and Petroleum Ether Extracts of Ocimum basilicum leaf. 2013;3:75-9.

How to cite this article: Mahata LE, Ali $\mathrm{H}$, Murni AW. Effect of streptozotocin on liver histology damage in rats model of gestational diabetes mellitus. International Journal of Research and Review. 2021; 8(9): 18-22. DOI: https://doi.org/10.52403/ijrr.20210904 